\title{
The Initial Mass Function of Be Stars
}

\author{
J. Zorec ${ }^{1}$, R. Levenhagen ${ }^{2}$, J. Chauville ${ }^{3}$, Y. Frémat ${ }^{3}$, D. Ballereau ${ }^{3}$, \\ A.M. Hubert ${ }^{3}$, M. Floquet $^{3}$, and N.V. Leister ${ }^{2}$ \\ ${ }^{1}$ Institut d'Astrophysique de Paris, UMR7095/CNRS, 98 bis bd. Arago, \\ F-75014 Paris, France, (zorec@iap.fr) \\ ${ }^{2}$ Instituto de Astronomia, Geofisica e Ciencias Atmosfericas, Sao Paulo, \\ Brazil \\ ${ }^{3}$ Observatoire de Paris-Meudon, GEPI, FRE/2459, 92195 Meudon, \\ France
}

\begin{abstract}
Allowing for systematic differences in the counting of Be stars due to their overluminosity, changes produced by their fast rotation on spectral types and time spent in the main sequence, a difference between the $\operatorname{IMF}(\mathrm{Be})$ and $\mathrm{IMF}(\mathrm{B})$ appears, which indicates that the appearance of the Be phenomenon may relay on differences in the initial star formation conditions.
\end{abstract}

\section{Motivation}

Several ways by which a star can present a Be phenomenon have been proposed so far: (1) accelerated stellar spinning in the secondary contraction phase (Cramping \& Hoyle 1960); (2) mass-transfer in close binary systems (Křiž \& Harmanec 1975); (3) innate stellar properties (Slettebak 1982); (4) the "Be mechanism" in the mass and angular momentum-gainer component in a close binary system after mass-transfer episode of class B (Packet 1981, Pols et al. 1991, Van Bever \& Vanbeveren 1997); (5) asynchronous critical rotation of a B component in a detached binary system (Harmanec et al. 2003). Zorec \& Briot (1997, ZB) concluded that the Be phenomenon may arise in whatever luminosity class, while Fabregat \& Torrejón have found that in open clusters it appears only in the second half of stellar main sequence life span. If the Be phenomenon responds to innate properties of stars due to their formation conditions, the IMF of Be stars should be different to that of B stars without emission lines.

\section{Method and Results}

We used B and Be stars from the Bright Stars Catalogue and Supplement. In a first step we assumed that: (a) the distribution in space of Be stars mirrors that of other B stars; (b) the relation between the visible absolute magnitude and mass is the same for both types of objects; (c) the main sequence lifetime of $\mathrm{B}$ and Be stars is the same; (d) the star formation rate is constant for each class of objects. The ratio between the IMF of B and Be stars is then given by the ratio of the respective present day mass functions. The magnitude-limited 


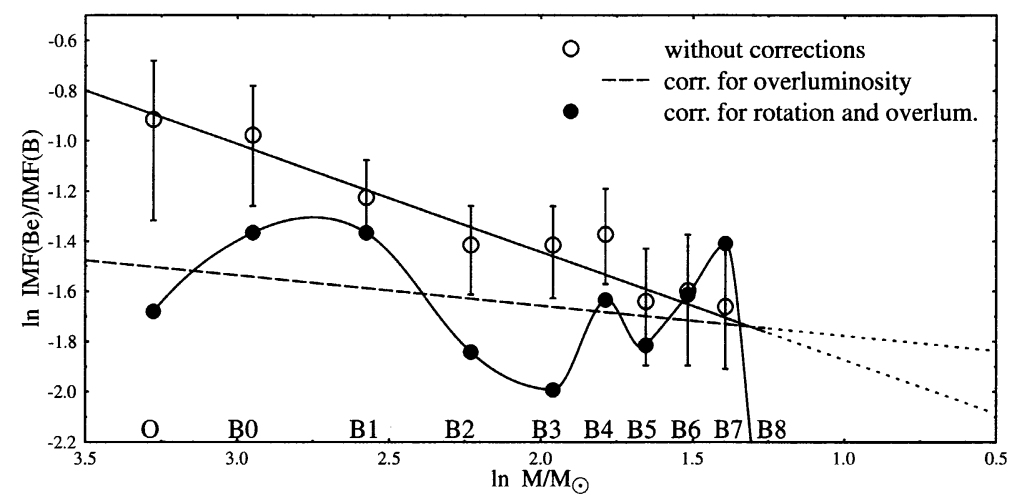

Figure 1. $\ln \mathrm{IMF}(\mathrm{Be}) / \mathrm{IMF}(\mathrm{B})$ against $\ln \mathrm{M} / \mathrm{M}_{\odot} . \circ$ : volume limited counts corrected for spectral classification uncertainties with its regression line; - - - : regression line of counts corrected from Be star overluminosity; $\bullet$ : counts corrected for Be overluminosity and rotation effects on spectral types and stellar ages

samples of $\mathrm{B}$ end Be stars were transformed into a volume-limited one. The $\ln \mathrm{N}(\mathrm{Be}) / \mathrm{N}(\mathrm{B})$ vs. $\ln \mathrm{M}$ thus obtained is shown as open circles in Fig.1. Owing to the circumstellar envelope, Be stars are brighter than $\mathrm{B}$ stars of same mass by $\Delta V \sim 0.0$ mag at $\mathrm{B} 8$ to $\Delta V \sim-(0.6-1.0)$ mag at $\mathrm{B} 0(\mathrm{ZB})$. The correction for this flux excess produces the distribution depicted by the dashed line in Fig. 1. Due to the fast rotation of Be stars, their spectral types are later than expected for their masses and their life times in the main sequence are longer (Meynet $\&$ Maeder 2000). These changes are not the same for all B subspectral type, mainly because the effects on spectral type changes are stronger the latest the B subspectral type. The ratio $\operatorname{IMF}(\mathrm{Be}) / \operatorname{IMF}(\mathrm{B})$ that is finally obtained after having taken all these effects is shown in Fig. 1 by full circles. Two main results are apparent in Fig. 1: (1) there is a strong difference between the IMF of B and Be stars, which implies that there may be some incidence of distictive initial formation conditions; 2) two rather well defined maxima appear around spectral types $\mathrm{B} 0-\mathrm{B} 1$ and $\mathrm{B} 7$.

\section{References}

Crampin J., Hoyle F. 1960, MNRAS 120, 33

Harmanec, P., Bisikalo, D.V., Boyarchuk, A.A. et al. 2003, A\&A 396, 937

Křiž S., \& Harmanec P. 1975, Bull. Astron. Inst. Czech. 26, 65

Meynet G., Maeder A. 2000, A\&A 361, 101

Packet W. 1981, A\&A 102, 17

Pols O.R., Coté J., Waters L.B.F.M. et al. 1991, A\&A 241, 419

Slettebak, A. 1982, ApJS 50, 55

Van Bever J., Vanbeveren D. 1997, A\&A 322, 116

Zorec J., Briot D. 1997, A\&A 318, 443 